\title{
Free Vibration Analysis of a Robotic Fish based on a Continuous and Non-uniform Flexible Backbone with Distributed Masses
}

William Coral , Claudio Rossi , Roque Saltaren , Oscar M Curet, and Antonio Barrientos

\begin{abstract}
This paper presents a Differential Quadrature Element Method for free transverse vibration of a robotic-fish based on a continuous and non-uniform flexible backbone with distributed masses (represented by ribs) based in the theory of a Timoshenko cantilever beam. The effects of the masses (Number, Magnitud and position) on the value of natural frequencies are investigated. Governing equations, compatibility and boundary conditions are formulated according to the Differential Quadrature rules. The compatibility conditions at the position of each distributed mass are assumed as the continuity in the vertical displacement, rotation and bending moment and discontinuity in the transverse force due to acceleration of the distributed mass. The convergence, efficiency and accuracy are compared to other analytical solutions proposed in the literature. Moreover, the proposed method has been validate against the physical prototype of a flexible fish backbone. The main advantages of this method, compared to the exact solutions available in the literature are twofold: first, smaller time-cost and second, it allows analysing the free vibration in beams whose section is an arbitrary function, which is normally difficult or even impossible with analytical other methods.
\end{abstract}

\section{Nomenclature}

$\begin{array}{cl}\xi & \text { Dimensionless Global Spatial Coordinate } \\ x & \text { Global Spatial Coordinate } \\ x^{(i)} & \text { Local Spatial Coordinate of Element } i \\ \lambda & \text { Dimensionless Natural Frequency of Vibration } \\ \omega & \text { Angular Natural Frequency of Vibration } \\ \psi(x, t), \Psi(x) & \text { Rotation Angle Due to Bending } \\ \rho & \text { Mass Density } \\ \vartheta & \text { Poissons Ratio } \\ A(x) & \text { Cross Sectional Area of the Beam } \\ E & \text { Youngs Modulus of Elasticity }\end{array}$




$\begin{array}{cl}G(x) & \text { Shear Modulus of Beam Material } \\ I(x) & \text { Moment of Inertia About the Neutral Axis } \\ J(x) & \text { Mass Moment of Inertia of the Beam per Unit Length } \\ k & \text { Shear Correction Factor } \\ L & \text { Total Length of the Beam } \\ m_{i} & \text { Translational Inertia of the } i^{t h} \text { Concentrated Mass } \\ N & \text { Number of Grid Points } \\ Q(x) & \text { Beam Shear Rigidity, } k G(x) A(x) \\ r & \text { Slender Ratio } \\ s & \text { Elastic Section Modulus } \\ t & \text { Time } \\ W^{(i)} & \text { Transverse Displacement of Element } i \\ \alpha_{i} & \text { Dimensionless Value of the } i \text { th Concentrated Attached Mass } \\ \zeta^{(i)} & \text { Dimensionless Local Spatial Coordinate of Element } i \\ \Psi^{(i)} & \text { Rotation Due to Bending of Element } i \\ A_{0} & \text { Values of the Cross-Section at the Clamped Edge } \\ I_{0} & \text { Values of the Moment of Inertia at the Clamped Edge } \\ l^{(i)} & \text { Dimensionless Length of Element } i \\ M^{(i)} & \text { Bending Moment in Element } i \\ V^{(i)} & \text { Transverse Force in Element } i \\ v^{(i)} & \text { Dimensionless Transverse Displacement of Element } i \\ w(x, t), W(x) & \text { Transverse Displacement }\end{array}$

\section{Introduction}

The literature of robotic fishes and bio-inspired robots has several examples of structures using flexible materials that bend to produce thrust, for maneuvering [4],[18], and even for energy harvesting [2]. However, few works analyze the normal frequency of vibration of the flexible structures employed as a way to maximize and optimize the use of energy. This allows a relatively small force applied repeatedly to make the amplitude of the oscillating system become very large. The purpose of this work is to study the resonance in structures to create a steady motion with low energy in order to create robots with improved energy efficiency. Concretely, we take into consideration a fish robot composed of a flexible backbone, made of polycarbonate, and a series of relatively heavy (i.e. whose weigh is non negligible) ribs (Fig. 1).

The dynamic characteristics of systems with flexible components is a very important issue that allows the study of robots based on a jointless structure. Some researchers have addressed the problem of vibration analysis of structures with distributed masses located at arbitrary positions using the Delta Dirac function [3], introducing the mass in the boundary conditions [12], using the Rayleigh-Ritz method [13]. or analyzing the case of flexible structures carrying distributed mass along the structure (including a free end) [8], [9], [16], [5]. In all cases such cases, the authors use the Bernoulli-Euller beam theory to model simple structures, which is reliable just for slender beams. In order to increase the accuracy and reliability of studies, especially for the beams with low length-to-thickness ratio, the study of the natural frequencies of a Timoshenko beam with a central point mass using coupled displacement field method has been proposed (see, e.g., [17]).

The Differential Quadrature Element Method (DQEM), provides a powerful numerical method to analyze the behavior (both static and dynamic) in structures with some discontinuities in loading, material properties or in its geometry. Thus, this method is applied to solve many problems especially in vibration analysis. 


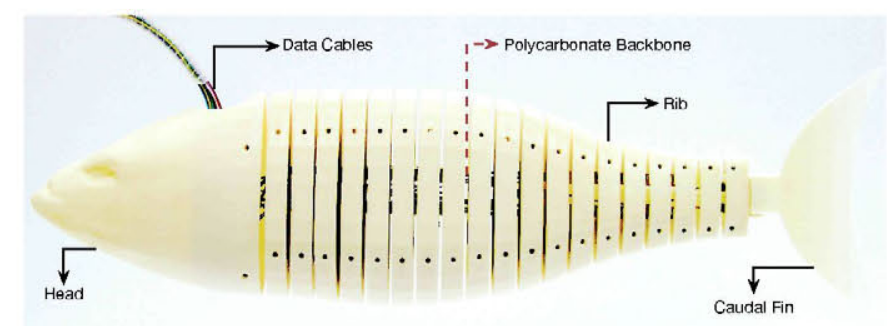

Fig. 1. The fish-robot prototype is composed of 19 ribs (excluding the head and the tail) made of 3D-printed ABS plastic that form the distributed masses.

\subsection{Structure of the paper}

In the following sections, we develop the method proposed for analyzing the vibrarion of a non-uniform cantilever with distributed masses. Initially the method adopts the Differential Quadrature Element Method (DQEM, see Section 2) to get high accuracy for estimating the boundary conditions in more than two points. This method is more efficient than the one presented by Timoshenko for each section along the beam. Then, in Section 3, governing equations are set for the free vibration using the Timoshenko beam. We don't use the Euler-Bernoulli beam theory because in this study the beam thickness is very small. Section 4 shows the accuracy of the proposed DQEM, which is verified by comparison with the exact solutions of the uniform Timoshenko beam presented by other researchers. Finally, we present an application of the proposed method to our concrete case.

\section{Differential quadrature method}

The Differential quadrature method allows expressing function derivatives in $x=x_{i}$ in terms of the value of function along the domain as:

$$
\left.\frac{d^{r} f}{d x^{r}}\right|_{x=x_{i}}=\sum_{j=1}^{N} A_{i j}{ }^{(r)} f_{j},
$$

where $A^{(r)}$ represents the weighting coefficient associated with the $r^{\text {th }}$ order derivative and $N$ the number of grid points [1]:

$$
\begin{aligned}
A_{i j}(1) & =\left\{\begin{array}{l}
\prod_{m=1}^{N}\left(x_{i}-x_{m}\right) \\
\frac{m \neq i, j}{\prod_{m=1}^{N}\left(x_{j}-x_{m}\right)},(i, j=1,2,3, \ldots N ; i \neq j) \\
m \neq j \\
\sum_{m=1}^{N} \frac{1}{\left(x_{i}-x_{m}\right)},(i=j=1,2,3, \ldots N) \\
A_{i j}(r)=\left\{\begin{array}{l}
r\left(A^{(r-1)}{ }_{i i} A^{(1)}{ }_{i j}-\frac{A^{(r-1)}}{x_{i}-x_{j}}\right),(i, j=1,2,3, \ldots N ; i \neq j) \\
-\sum_{m=1}^{N} A^{(r)}{ }_{i m},(i=j=1,2,3, \ldots N)
\end{array}\right. \\
m \neq i
\end{array}\right.
\end{aligned}
$$

Using a set of grid points (blue dots pot Fig. 2) following Gauss-Lobatto-Chebyshev points for interval $[0,1]$ we have

$$
\bar{x}_{i}=\frac{1}{2}\left\{1-\cos \left[\frac{(i-1) \pi}{(N-1)}\right]\right\},(i=1,2,3, \ldots N) .
$$




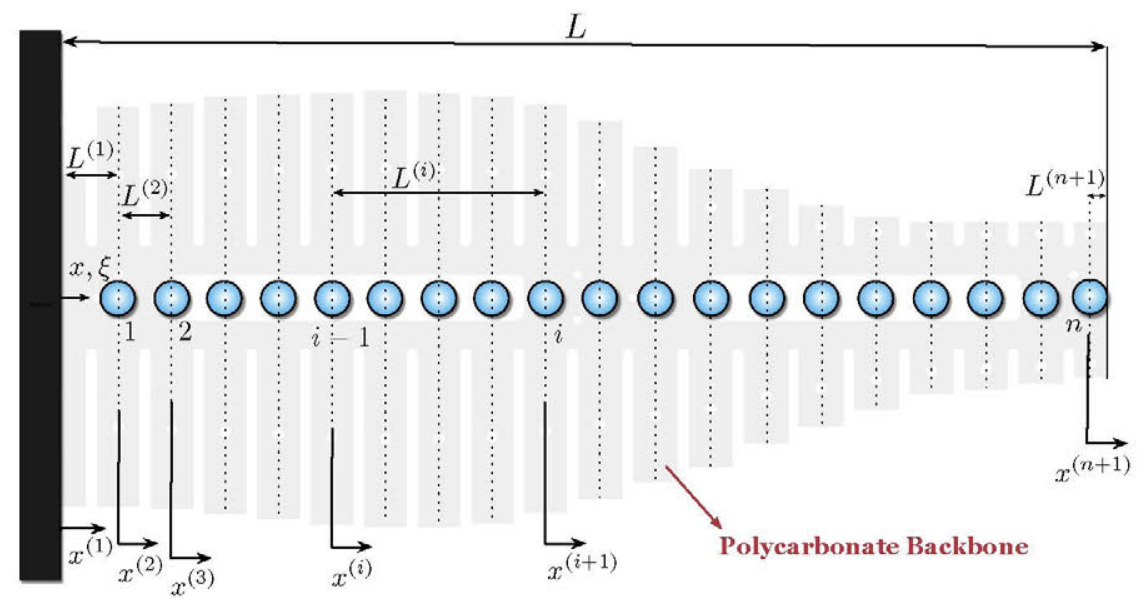

Fig. 2. Non-uniform fish-robot backbone with distributed masses.

This set of grid point shows the compression of the two end points in the interval [0,1], providing in this way high accuracy for estimating the value of the derivative of the function at the boundary points.

\section{Vibration analysis}

\subsection{Governing equations}

Figure 2 shows the fish-robot backbone. Note that the mass of each rib is different and the backbone is non-uniform. The entire surface is modelled like a nonuniform cantilever Timoshenko beam. The Free Vibration governing equations for a Timoshenko beam with distributed masses are written as [20]:

$$
\begin{aligned}
& \frac{\partial}{\partial x}\left\{k G A(x)\left[\frac{\partial w(x, t)}{\partial x}-\psi(x, t)\right]\right\}-\rho A(x) \frac{\partial^{2} w(x, t)}{\partial t^{2}}=0 \\
& \frac{\partial}{\partial x}\left[E I(x) \frac{\partial \psi(x, t)}{\partial x}\right]+k G A(x)\left[\frac{\partial w(x, t)}{\partial x}-\psi(x, t)\right]-\rho I(x) \frac{\partial^{2} w(x, t)}{\partial t^{2}}=0
\end{aligned}
$$

where $w(x, t)$ is vertical displacement. The term $k$ is introduced to take into account the geometry dependent distribution of the shear stress and depends on the shape of the section and the Poisson ratio of the material [10]. The displacement $w(x, t)$ and rotation $\psi(x, t)$ can be assumed as the product of the functions $W(x)$ and $\Psi(x)$ which only depend on the spatial coordinate $x$ and a time dependent harmonic function as

$$
w(x, t)=W(x) e^{i \omega t} \quad \psi(x, t)=\Psi(x) e^{i \omega t}
$$

Substituting the Equations 5 into the set of Equations 4, we obtain

$$
\begin{aligned}
& \frac{d^{2} W(x)}{d x^{2}}-\frac{d \Psi(x)}{d x}+\frac{1}{A^{*}(x)} \frac{d A^{*}(x)}{d x}\left[\frac{d W(x)}{d x}-\Psi(x)\right]+\frac{\rho \omega^{2}}{k G} W(x)=0, \\
& \frac{E I_{0}}{k A_{0} G}\left[\frac{d^{2} \Psi(x)}{d x^{2}}+\frac{1}{I^{*}(x)} \frac{d I^{*}(x)}{d x} \frac{d \Psi(x)}{d x}\right]+\frac{A^{*}(x)}{I^{*}(x)}\left[\frac{d W(x)}{d x}-\Psi(x)\right]+\frac{\rho I_{0} \omega^{2}}{k A_{0} G} \Psi(x)=0
\end{aligned}
$$

The second moment of inertia and cross-sectional area are written in the following dimensionless form:

$$
I^{*}=\frac{I(x)}{I_{0}} \quad A^{*}(x)=\frac{A(x)}{A_{0}}
$$


where $I_{0}$ and $A_{0}$ are values of the moment of inertia and cross-section at the clamped edge of the beam. For the $i^{\text {th }}$ sub-beam, the set of Eqs. 6 are written as

$$
\begin{aligned}
& \frac{d^{2} W^{(i)}\left(x^{(i)}\right)}{d\left(x^{(i)}\right)}-\frac{d \Psi^{(i)}\left(x^{(i)}\right)}{d x^{(i)}}+\frac{1}{A^{*}(x)} \frac{d A^{*}(x)}{d x}\left[\frac{d W^{(i)}\left(x^{(i)}\right)}{d x^{(i)}}-\Psi^{(i)}\left(x^{(i)}\right)\right]+\frac{\rho \omega^{2}}{k G} W^{(i)}\left(x^{i}\right)=0 \\
& \frac{E I_{0}}{k A_{0} G}\left[\frac{d^{2} \Psi^{(i)}\left(x^{(i)}\right)}{d\left(x^{(i)}\right)^{2}}+\frac{1}{I^{*}(x)} \frac{d I^{*}(x)}{d x} \frac{d \Psi^{(i)}\left(x^{(i)}\right)}{d x^{(i)}}\right]+\frac{A^{*}(x)}{I^{*}(x)}\left[\frac{d W^{(i)}\left(x^{(i)}\right)}{d x^{(i)}}-\Psi^{(i)}\left(x^{(i)}\right)\right] \\
& +\frac{\rho I_{0} \omega^{2}}{k A_{0} G} \Psi^{(i)}\left(x^{(i)}\right)=0
\end{aligned}
$$

Introducing the dimensionless parameters:

$$
\xi=\frac{x}{L} \quad \zeta^{(i)}=\frac{x^{(i)}}{L^{(i)}} \quad v^{(i)}=\frac{W^{(i)}}{L} \quad l^{(i)}=\frac{L^{(i)}}{L}
$$

the set of Eqs. 8 can be rewritten as

$$
\begin{gathered}
\left(\frac{1}{l(i)}\right)^{2} \frac{d^{2} v^{(i)}\left(\zeta^{(i)}\right)}{d\left(\zeta^{(i)}\right)^{2}}-\left(\frac{1}{l^{(i)}}\right) \frac{d \Psi^{(i)}\left(\zeta^{(i)}\right)}{d \zeta^{(i)}}+\frac{1}{A^{*}(\xi)} \frac{d A^{*}(\xi)}{d \xi}\left[\left(\frac{1}{l^{(i)}}\right) \frac{d v^{(i)}\left(\zeta^{(i)}\right)}{d \zeta^{(i)}}-\Psi^{(i)}\left(\zeta^{(i)}\right)\right] \\
+\lambda^{4} s^{2} v^{(i)}\left(\zeta^{(i)}\right)=0
\end{gathered}
$$

where

$$
\lambda^{4}=\frac{\rho A_{0} L^{4} \omega^{2}}{E I_{0}}, \quad s^{2}=\frac{E I_{0}}{k A_{0} G L^{2}}=\frac{2(1+v)}{k} r^{2}, \quad r^{2}=\frac{I_{0}}{A_{0} L^{2}} .
$$

Assuming all grid points are the same for the sub-beams, then

$$
\zeta^{(1)}=\zeta^{(2)}=\zeta^{(3)}=\ldots=\zeta^{(i)}=\ldots=\zeta^{(n+1)}=\zeta .
$$

Thus, Eq. 10 can be simplified:

$$
\begin{aligned}
& \left(\frac{1}{l^{(i)}}\right)^{2} \frac{d^{2} v^{(i)}(\zeta)}{d \zeta^{2}}-\left(\frac{1}{l^{(i)}}\right) \frac{d \Psi^{(i)}(\zeta)}{d \zeta}+\frac{1}{A^{*}(\xi)} \frac{d A^{*}(\xi)}{d \xi}\left[\left(\frac{1}{l^{(i)}}\right) \frac{d v^{(i)}(\zeta)}{d(\zeta)}-\Psi^{(i)}(\zeta)\right] \\
& +\lambda^{4} s^{2} v^{(i)}(\zeta)=0 \\
& s^{2}\left[\left(\frac{1}{l^{(i)}}\right)^{2} \frac{d^{2} \Psi^{(i)}(\zeta)}{d \zeta^{2}}+\frac{1}{I^{*}(\xi)} \frac{d I^{*}(\xi)}{d \xi}\left(\frac{1}{l^{(i)}}\right) \frac{d \Psi^{(i)}(\zeta)}{d \zeta}\right]+\frac{A^{*}(\xi)}{I^{*}(\xi)}\left[\left(\frac{1}{l^{(i)}}\right) \frac{d v^{(i)}(\zeta)}{d(\zeta)}-\Psi^{(i)}(\zeta)\right]
\end{aligned}
$$

Furthermore, we introduce a modified form of the weighting coefficients of element $i$ to simplify the DQ analogue equations defined as

$$
[A]^{(i)}=\frac{[A]^{(i)}}{l^{(i)}} \quad[B]^{(i)}=\frac{[A]^{(i)}}{\left(l^{(i)}\right)^{2}}
$$

Using Eq. 14, we obtain for the governing set of equations of element $i$ the DQ analogue:

$$
\begin{aligned}
& {\left[B_{v e}\right]^{(i)}\{v\}^{(i)}-\left[A_{s e}\right]^{(i)}\{\Psi\}^{(i)}+\lambda^{4} s^{2}\{v\}^{(i)}=0,} \\
& {\left[B_{s e}\right]^{(i)}\{\Psi\}^{(i)}-\left[A_{v e}\right]^{(i)}\{v\}^{(i)}+\lambda^{4} s^{2} r^{2}\{\Psi\}^{(i)}=0}
\end{aligned}
$$

where

$$
\begin{aligned}
& {\left[B_{v e}\right]^{(i)}=[B]^{(i)}+\left[\frac{1}{A^{*}(\xi)} \frac{d A^{*}(\xi)}{d \xi}\right]^{(i)}[A]^{(i)} \quad\left[A_{v e}\right]^{(i)}=\left[\frac{A^{*}(\xi)}{I^{*}(\xi)}\right]^{(i)}[A]^{(i)}} \\
& {\left[B_{s e}\right]^{(i)}=s^{2}\left([B]^{(i)}+\left[\frac{1}{I^{*}(\xi)} \frac{d I^{*}(\xi)}{d \xi}\right]^{(i)}[A]^{(i)}\right)-\left[\frac{A^{*}(\xi)}{I^{*}(\xi)}\right]^{(i)}} \\
& {\left[A_{s e}\right]^{(i)}=[A]^{(i)}+\left[\frac{1}{A^{*}(\xi)} \frac{d A^{*}(\xi)}{d \xi}\right]^{(i)} .}
\end{aligned}
$$

In Eq. 16, the terms

$$
\left[\frac{1}{A^{*}(\xi)} \frac{d A^{*}(\xi)}{d \xi}\right]^{(i)}, \quad\left[\frac{A^{*}(\xi)}{I^{*}(\xi)}\right]^{(i)}, \quad\left[\frac{1}{I^{*}(\xi)} \frac{d I^{*}(\xi)}{d \xi}\right]^{(i)}
$$


are geometry-dependent diagonal matrices with values of the geometrical parameters. Now we can rewrite the motion equations 15 for the domain points in order to eliminate the redundant equations ([6],[7] [15]) obtaining:

$$
\begin{aligned}
& \left.B_{v e}\right]^{(i)}\{v\}^{(i)}-A_{s e}{ }^{(i)}\{\Psi\}^{(i)}+\lambda^{4} s^{2}\{\bar{v}\}^{(i)}=0 \\
& {\left[B_{s e}\right]^{(i)}\{\Psi\}^{(i)}+\left[A_{v e}\right]^{(i)}\{v\}^{(i)}+\lambda^{4} s^{2} r^{2}\{\bar{\Psi}\}^{(i)}=0}
\end{aligned}
$$

where bar signs means truncated non-square matrices. Combining equations Eqs.17:

$$
\left[B_{v}\right]\{v\}-\left[A_{s}\right]\{\Psi\}+\lambda^{4} s^{2}\{v\}_{d}=0 \quad\left[B_{s}\right]\{\Psi\}+\left[A_{v}\right]\{v\}+\lambda^{4} s^{2} r^{2}\{\Psi\}_{d}=0
$$

where

$$
\begin{aligned}
& \{v\}=\left\{\left\{\begin{array}{c}
v_{1}{ }^{(1)} \\
v_{2}{ }^{(1)} \\
\cdot \\
\vdots \\
v_{N-1}(1) \\
v_{N}{ }^{(1)}
\end{array}\right\}^{T}\left\{\begin{array}{c}
v^{(2)} \\
v_{2}{ }^{(2)} \\
\vdots \\
\vdots \\
v_{N-1}{ }^{(2)} \\
v_{N}{ }^{(2)}
\end{array}\right\}^{T} \cdots\left\{\begin{array}{c}
v_{1}{ }^{(n+1)} \\
v_{2}{ }^{(n+1)} \\
\vdots \\
\vdots \\
v_{N-1}{ }^{(n+1)} \\
v_{N}{ }^{(n+1)}
\end{array}\right\}^{T}\right\}^{T}
\end{aligned}
$$

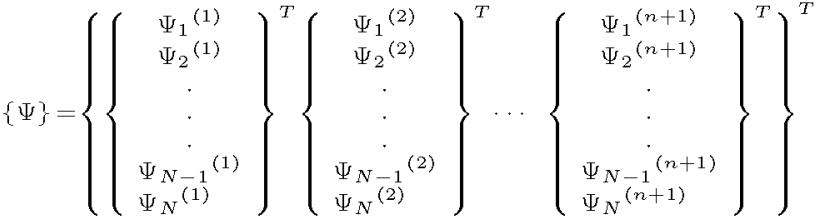

$$
\begin{aligned}
& {\left[B_{v}\right]=\operatorname{diag}\left(\left[\overline{B_{v e}}\right]^{(1)}\left[\overline{B_{v e}}\right]^{(2)} \ldots\left[\overline{B_{v e}}\right]^{(n+1)}\right)\left[A_{v}\right]=\operatorname{diag}\left(\left[\overline{A_{v e}}\right]^{(1)}\left[\overline{A_{v e}}\right]^{(2)} \ldots\left[\overline{A_{v e}}\right]^{(n+1)}\right)} \\
& {\left[B_{s}\right]=\operatorname{diag}\left(\left[\overline{B_{s e}}\right]^{(1)}\left[\overline{B_{s e}}\right]^{(2)} \cdots\left[\overline{B_{s e}}\right]^{(n+1)}\right) \quad\left[A_{s}\right]=\operatorname{diag}\left(\left[\overline{A_{s e}}\right]^{(1)}\left[\overline{A_{s e}}\right]^{(2)} \cdots\left[\overline{A_{s e}}\right]^{(n+1)}\right)}
\end{aligned}
$$

The "diag" operator provides the diagonal matrices. In order to separate the domain, boundary, adjacent displacement and rotation components, Equations (18) need to be rearranged $[11]$ :

$$
\begin{aligned}
& {\left[B_{v}\right]_{b}\{v\}_{b}+\left[B_{v}\right]_{d}\{v\}_{d}+\left[B_{v}\right]_{c}\{v\}_{c}-\left[A_{s}\right]_{b}\{\Psi\}_{b}-\left[A_{s}\right]_{d}\{\Psi\}_{d}-\left[A_{s}\right]_{c}\{\Psi\}_{c}+\lambda^{4} s^{2}\{v\}_{d}=0} \\
& {\left[A_{v}\right]_{b}\{v\}_{b}+\left[A_{v}\right]_{d}\{v\}_{d}+\left[A_{v}\right]_{c}\{v\}_{c}+\left[B_{s}\right]_{b}\{\Psi\}_{b}+\left[B_{s}\right]_{d}\{\Psi\}_{d}+\left[B_{s}\right]_{c}\{\Psi\}_{c}+\lambda^{4} s^{2} r^{2}\{\Psi\}_{d}=0}
\end{aligned}
$$

where

$$
\begin{aligned}
& \left\{v_{f_{b}}=\left\{\begin{array}{c}
\left\{v_{1}^{(1)}\right\} \\
\left\{v_{N}^{(n+1)}\right.
\end{array}\right\}\left\{\left\{\Psi_{\}_{b}}=\left\{\begin{array}{c}
\left\{\Psi_{1}^{(1)}\right\} \\
\left\{\Psi_{N}^{(n+1)}\right.
\end{array}\right\}\right\}\right.\right. \\
& \{v\}_{c}=\left\{\left\{v_{N}{ }^{(1)}\right\}\left\{\begin{array}{c}
v_{1}^{(2)} \\
v_{N}(2)
\end{array}\right\}^{T}\left\{\begin{array}{c}
v_{1}^{(3)} \\
v_{N}(3)
\end{array}\right\}^{T} \cdots\left\{\begin{array}{c}
v_{1}(n) \\
v_{N}(n)
\end{array}\right\}^{T}\left\{v_{1}^{(n+1)}\right\}\right\}^{T} \\
& \{\Psi\}_{c}=\left\{\left\{\Psi_{N}^{(1)}\right\}\left\{\begin{array}{c}
\Psi_{1}^{(2)} \\
\Psi_{N}(2)
\end{array}\right\}^{T}\left\{\begin{array}{c}
\Psi_{1}^{(3)} \\
\Psi_{N}(3)
\end{array}\right\}^{T} \cdots\left\{\begin{array}{c}
\Psi_{1}^{(n)} \\
\Psi_{N}(n)
\end{array}\right\}^{T}\left\{\Psi_{1}(n+1)\right\}\right\}^{T} \\
& \{v\}_{d}=\left\{\left\{\begin{array}{c}
v_{2}{ }^{(1)} \\
\vdots \\
v_{N-1}{ }^{(1)}
\end{array}\right\}^{T}\left\{\begin{array}{c}
v_{2}{ }^{(2)} \\
\vdots \\
v_{N-1}{ }^{(2)}
\end{array}\right\}^{T} \cdots\left\{\begin{array}{c}
v_{2}{ }^{(n+1)} \\
\vdots \\
v_{N-1}(n+1)
\end{array}\right\}^{T}\right\}^{T} \\
& \left\{\Psi_{d}=\left\{\left\{\begin{array}{c}
\Psi_{2}^{(1)} \\
\vdots \\
\Psi_{N-1}{ }^{(1)}
\end{array}\right\}^{T}\left\{\begin{array}{c}
\Psi_{2}{ }^{(2)} \\
\vdots \\
\Psi_{N-1}{ }^{(2)}
\end{array}\right\}^{T} \cdots\left\{\begin{array}{c}
\Psi_{2}^{(n+1)} \\
\vdots \\
\Psi_{N-1}{ }^{(n+1)}
\end{array}\right\}^{T}\right\}^{T}\right.
\end{aligned}
$$

\subsection{Compatibility conditions}

In the following, we analyse the compatibility conditions that link the inertia and elasticity of the beam with the distributes masses. 
Around of each concentrated mass, effects $\left[x_{m}^{-}, x_{m}^{+}\right]$, neglecting the moment of inertia of the concentrated mass, the compatibility conditions are continuous in the vertical displacement and rotation due to the acceleration of distributed masses that produce a bending moment and alike discontinuous in the transverse force.

$$
\begin{aligned}
& w\left(x_{m}^{-}, t\right)=w\left(x_{m}^{+}, t\right) \quad \psi\left(x_{m}^{-}, t\right)=\psi\left(x_{m}^{+}, t\right) \quad M\left(x_{m}^{-}, t\right)=M\left(x_{m}^{+}, t\right) \\
& V\left(x_{m}^{-}, t\right)-V\left(x_{m}^{+}, t\right)=m_{i} \frac{\partial^{2} w(x, t)}{\partial t^{2}}
\end{aligned}
$$

where $m_{i}, M, V$ are the translational inertia of the $i^{\text {th }}$ concentrated mass, the bending moment and shear force respectively, which are presented for $i^{\text {th }}$ sub-beam as [20]

$$
M^{(i)}=E I \frac{d \Psi^{(i)}}{d x^{(i)}}=\frac{E I}{L} \frac{1}{l^{(i)}} \frac{d \Psi^{(i)}}{d \zeta} \quad V^{(i)}=k A G\left(\Psi^{(i)}-\frac{d W^{(i)}}{d x^{(i)}}\right)=k A G\left(\Psi^{(i)}-\frac{1}{l^{(i)}} \frac{d \Psi^{(i)}}{d \zeta}\right)
$$

Compatibility Conditions can be expressed in the DQ form as

$$
\begin{aligned}
& v_{N}^{(i)}=v_{1}^{(i+1)} \quad \Psi_{N}^{(i)}=\Psi_{1}^{(i+1)} \sum_{j=1}^{N} A_{1 j}^{(i+1)} \Psi_{j}^{(i+1)}-\sum_{j=1}^{N} A_{N j}^{(i)} \Psi_{j}^{(i)}=0 \\
& \sum_{j=1}^{N} A_{1 j}^{(i+1)} v_{j}^{(i+1)}-\sum_{j=1}^{N} A_{N j}^{(i)} v_{j}^{(i)}+\frac{\alpha_{i} s^{2} \lambda^{4}}{A^{*}\left(\xi_{i}\right)} v_{N}^{(i)}=0
\end{aligned}
$$

we can define the dimensionless translational inertias of the $i^{\text {th }}$ concentrated mass as

$$
\alpha_{i}=\frac{m_{i}}{\rho A_{0} L}
$$

Eq.24 can be rewritten in matrix form as

$$
\left[Q_{e}\right]^{(i)}\left\{\begin{array}{c}
\{v\}^{(i)} \\
\{v\}^{(i+1)}
\end{array}\right\}+\lambda^{4}\left[q_{e}\right]^{(i)}\left\{\begin{array}{c}
\{v\}^{(i)} \\
\{v\}^{(i+1)}
\end{array}\right\}=\left\{\begin{array}{l}
0 \\
0
\end{array}\right\} \quad\left[Q_{e}\right]^{(i)}\left\{\begin{array}{c}
\{\Psi\}^{(i)} \\
\{\Psi\}^{(i+1)}
\end{array}\right\}=\left\{\begin{array}{l}
0 \\
0
\end{array}\right\}
$$

where

$$
\begin{aligned}
& {\left[Q_{e}\right]_{j k}^{(i)}= \begin{cases}-\delta_{N K} & j=1,1 \leq k \leq N \\
\delta_{(N+1) K} & j=1, N+1 \leq k \leq 2 N \\
-A_{N k}^{(i)} & j=2,1 \leq k \leq N \\
A_{1 k}^{(i+1)} & j=2, N+1 \leq k \leq 2 N\end{cases} } \\
& j=1,2 \\
& 1 \leq k \leq 2 N \\
& {\left[q_{e}\right]_{j k}^{(i)}= \begin{cases}\frac{\alpha_{i} s^{2}}{A^{*}\left(\xi_{i}\right)} & j=2, k=N \\
0 & \text { else }\end{cases} }
\end{aligned}
$$

Rewriting and composing a new Eq.26 for all sub-beams,

$$
[Q]\{v\}+\lambda^{4}[q]\{v\}=\{0\} \quad[Q]\{\Psi\}=\{0\}
$$

where $[Q],[q]$ are corresponding matrix that contains $\left[Q_{e}\right]^{(1)}$ to $\left[Q_{e}\right]^{(n+1)}$ and $\left[q_{e}\right]^{(1)}$ to $\left[q_{e}\right]^{(n+1)}$ respectively. Eq.26 may be rewritten and sectioned to separate the components of domain, boundary, and adjacent displacement

$$
\begin{aligned}
& {[Q]_{b}\{v\}_{b}+[Q]_{d}\{v\}_{d}+[Q]_{c}\{v\}_{c}+\lambda^{4}\left([q]_{b}\{v\}_{b}+[q]_{d}\{v\}_{d}+[q]_{c}\{v\}_{c}\right)=\{0\}} \\
& {[Q v]_{b}\{\Psi\}_{b}+[Q v]_{d}\{\Psi\}_{d}+[Q v]_{c}\{\Psi\}_{c}=\{0\}}
\end{aligned}
$$

From Eq.27, it cab be concluded that $[q]_{b}=[q]_{d}=0$. Therefore, Eq.29 can be summarized as

$$
\begin{gathered}
{[Q]_{b}\{v\}_{b}+[Q]_{d}\{v\}_{d}+[Q]_{c}\{v\}_{c}+\lambda^{4}[q]_{c}\{v\}_{c}=\{0\}} \\
\{\Psi\}_{c}=\left[J_{b}\right]\{\Psi\}_{b}+\left[J_{d}\right]\{\Psi\}_{d}
\end{gathered}
$$

where

$$
\left[J_{b}\right]=-\left[Q_{v}\right]_{c}^{-1}\left[Q_{v}\right]_{b} \quad\left[J_{d}\right]=-\left[Q_{v}\right]_{c}^{-1}\left[Q_{v}\right]_{d}
$$

Substituting Eq.31 into Eq.20

$$
\begin{aligned}
& {\left[B_{v}\right]_{b}\{v\}_{b}+\left[B_{v}\right]_{d}\{v\}_{d}+\left[B_{v}\right]_{c}\{v\}_{c}+\left[G_{s b}\right]\{\Psi\}_{b}+\left[G_{s d}\right]\{\Psi\}_{d}+\lambda^{4} s^{2}\{v\}_{d}=\{0\}} \\
& {\left[A_{v}\right]_{b}\{v\}_{b}+\left[A_{v}\right]_{d}\{v\}_{d}+\left[A_{v}\right]_{c}\{v\}_{c}+\left[E_{s b}\right]\{\Psi\}_{b}+\left[E_{s d}\right]\{\Psi\}_{d}+\lambda^{4} s^{2} r^{2}\{\Psi\}_{d}=\{0\}}
\end{aligned}
$$

where

$$
\begin{aligned}
& {\left[G_{s b}\right]=\left[A_{s}\right]_{b}+\left[A_{s}\right]_{c}\left[J_{b}\right] \quad\left[G_{s d}\right]=\left[A_{s}\right]_{d}+\left[A_{s}\right]_{c}\left[J_{d}\right] \quad\left[E_{s b}\right]=\left[B_{s}\right]_{b}+\left[B_{s}\right]_{c}\left[J_{b}\right]} \\
& {\left[E_{s d}\right]=\left[B_{s}\right]_{d}+\left[B_{s}\right]_{c}\left[J_{d}\right]}
\end{aligned}
$$




\subsection{Boundary conditions}

The boundary conditions for a cantilever beam (robot fish backbone) depicted in Fig.2 can be considered as

$$
\left.W^{(1)}\right|_{x^{(1)}=0}=\left.0 \quad \psi^{(1)}\right|_{x^{(1)}=0}=\left.0 \quad V^{(n+1)}\right|_{x^{(n+1)}=L^{(n+1)}}=\left.0 \quad M^{(n+1)}\right|_{x^{(n+1)}=L^{(n+1)}}=0
$$

Eq.35 can be rewritten using Eq.23 as

$$
\left.\Psi^{(1)}\right|_{x^{(1)}=0}=\left.0 \quad \frac{1}{l^{(i)}} \frac{d \Psi(n+1)}{d \zeta}\right|_{\zeta=1}=\left.0 \quad v^{(1)}\right|_{x^{(1)}=0}=\left.0 \quad\left(\frac{1}{l^{(i)}} \frac{d \Psi(n+1)}{d \zeta}-\Psi^{(n+1)}\right)\right|_{\zeta=1}=0
$$

In the DQ form, Eq. (36) can be indicated as

$$
\begin{gathered}
{[m]\{\Psi\}=0} \\
{[m]\{v\}+[n]\{\Psi\}=\{0\}}
\end{gathered}
$$

where

$$
\begin{aligned}
& m_{j k}= \begin{cases}1 & j=k=1 \\
A_{N(k-n N)}^{(n+1)} & j=2, n N+1 \leq k \leq(n+1) N \\
0 & \text { else }\end{cases} \\
& j=1,2 \\
& 1 \leq k \leq(n+1) N \\
& n_{j k}= \begin{cases}-1 & j=2, k=(n+1) N \\
0 & \text { else }\end{cases}
\end{aligned}
$$

Eqs.37 and 38 can be rewritten and sectioned in order to separate the components (boundary, domain, adjacent displacement and rotation)

$$
\begin{aligned}
& {[m]_{b}\{\Psi\}_{b}+[m]_{d}\{\Psi\}_{d}+[m]_{c}\{\Psi\}_{c}=\{0\}} \\
& {[m]_{b}\{v\}_{b}+[m]_{d}\{v\}_{d}+[m]_{c}\{v\}_{c}+[n]_{d}\{\Psi\}_{d}+[n]_{c}\{\Psi\}_{c}=\{0\}}
\end{aligned}
$$

From Eq.39, we know that $[n]_{c}=[n]_{d}=0$; Hence, using Eq.31, Eq.40 we have

$$
\{\Psi\}_{b}=[t]\{\Psi\}_{d} \quad\{v\}_{b}=-[m]_{b}^{-1}[m]_{d}^{-1}\{v\}_{d}-[m]_{b}^{-1}[m]_{c}^{-1}\{v\}_{c}-[m]_{b}^{-1}[n]_{b}[t]\{\Psi\}_{d}
$$

where

$$
[t]=-[r]_{b}^{-1}[r]_{d} \quad[r]_{b}=[m]_{b}+[m]_{c}\left[J_{b}\right] \quad[r]_{b}=[m]_{d}+[m]_{c}\left[J_{d}\right]
$$

Replacing Eq.41 into the set of Eqs.20 and 30, a new set of equations is obtained

$$
[K]\left\{\begin{array}{c}
\{v\}_{d} \\
\{v\}_{c} \\
\{\Psi\}_{d}
\end{array}\right\}=\lambda^{4}[M]\left\{\begin{array}{c}
\{v\}_{d} \\
\{v\}_{c} \\
\{\Psi\}_{d}
\end{array}\right\}
$$

where

$$
\begin{aligned}
& {[K]=} {\left[\begin{array}{ccc}
{\left[B_{v}\right]_{d}-\left[B_{v}\right]_{b}[m]_{b}^{-1}[m]_{d}} & {\left[B_{v}\right]_{c}-\left[B_{v}\right]_{b}[m]_{b}^{-1}[m]_{c}} & {\left[G_{s d}\right]+\left[G_{s b}\right][t]-\left[B_{v}\right]_{b}[m]_{b}^{-1}[n]_{b}[t]} \\
{[Q]_{d}-[Q]_{b}[m]_{b}^{-1}[m]_{d}} & {[Q]_{c}-[Q]_{b}[m]_{b}^{-1}[m]_{c}} & -[Q]_{b}[m]_{b}^{-1}[n]_{b}[t] \\
{\left[A_{v}\right]_{d}-\left[A_{v}\right]_{b}[m]_{b}^{-1}[m]_{d}} & {\left[A_{v}\right]_{c}-\left[A_{v}\right]_{b}[m]_{b}^{-1}[m]_{c}} & {\left[E_{s d}\right]+\left[E_{s b}\right][t]-\left[A_{v}\right]_{b}[m]_{b}^{-1}[n]_{b}[t]}
\end{array}\right] } \\
& M=-\left[\begin{array}{ccc}
s^{2} I_{(n+1)(N-2) *(n+1)(N-2)} & \{0\}_{(n+1)(N-2) * 2 n} & \{0\}_{(n+1)(N-2) *(n+1)(N-2)} \\
\{0\}_{2 n *(n+1)(N-2)} & {[q]_{c}} & \{0\}_{2 n *(n+1)(N-2)} \\
\{0\}_{(n+1)(N-2) *(n+1)(N-2)} & \{0\}_{(n+1)(N-2) * 2 n} s^{2} r^{2} I_{(n+1)(N-2) *(n+1)(N-2)}
\end{array}\right]
\end{aligned}
$$

Using Eq.44, we can determine the natural frequencies and corresponding mode shapes. The corresponding mode shapes can be completed using the Eqs.31 and 41. It should be noted that the number of grid points affects the results. The number of grid points must be determined to satisfy the following relation for convergence of first $n$ frequencies:

$$
\left|\frac{\lambda_{l}{ }^{(N)}-\lambda_{l}{ }^{(N-1)}}{\lambda_{l}{ }^{(N-1)}}\right| \leq \varepsilon \quad l=1,2, \ldots n
$$

where $\varepsilon$ is considered as 0.01 in this study. 


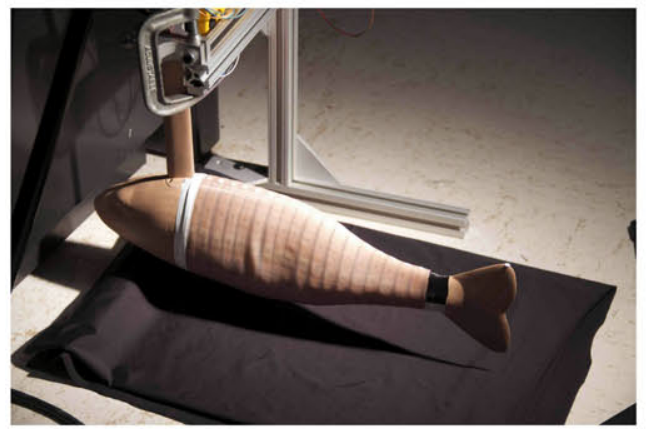

Fig. 3. Robot-Fish including a Silicone-rubber-based skin

\section{Experimental results}

\subsection{Numerical comparison}

In order to assess the effectiveness of the proposed method, we have compared its accuracy with the exact solution obtained with the method proposed by Lee and Len [14].

We applied both methods to a cantilever Timoshenko beam with $(v=0.25, k=2 / 3)$, an attached tip mass $(\alpha=0.32)$, beam cross-sectional properties $A=A_{0}(1-0.4 \xi)$ and $I=I_{0}(1-0.4 \xi)^{3}$ for a slenderness ratio $r$ of 0.1 and 0.04 as shown in Table.1. Also it can be observed the good accuracy for the proposed method.

Table 1. Comparison between this method and the exact method proposed by [14] for the first three non-dimensional frequencies $\left(\lambda^{2}\right)$

\begin{tabular}{lllllll}
\hline Slenderness ratios $r$ & & 0.04 & & \multicolumn{3}{l}{0.1} \\
\hline Modes of Vibration & 1 & 2 & 3 & 1 & 2 & 3 \\
\hline Presented Method & 2.117 & 13.42 & 36.11 & 1.997 & 10.69 & 24.34 \\
Lee and Lin (1995) & 2.099 & 13.55 & 36.76 & 2.015 & 11.07 & 25.63 \\
\hline
\end{tabular}

\subsection{Practical application}

Our ultimate purpose was to analyze the natural frequency in the robot-fish we are developing, in order to optimize its energy efficiency. Therefore, we have applied our method to the physical prototype using the setup depicted Fig. 3. The parameters modelling the robot fish body as a conical Timoshenko beam where: slender ratio $(r=1.03)$, elastic section modulus $(s=2.58)$, diameter variation $d=d_{0}(1-0.5 \xi)$ and uniformly spaced distributed masses $(\alpha=1.3)$. The number of distributed masses $(n)$ are 19. The first three modes are depicted in Fig.6

In order to analyse the behavior of the backbone, the tail was set to its inicial position and then released. Fig. 4 shows the evolution of this movement. A complete tail beat lasted 6 seconds. White marks in the tail were used to allow a particle tracking software to find the free vibration response (Fig. 5a) and the experimental Natural Frequency (Fig. 5b), using a camera to capture the movement at 60 frames per second.

From the analysis of the images recorded, the natural frequency of the structure obtained experimentally was 1.873 . 


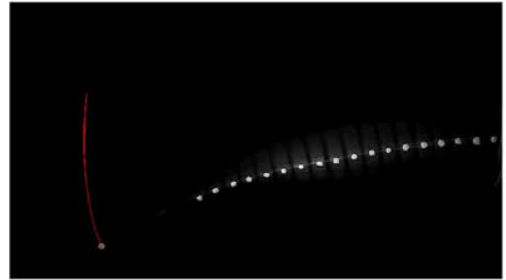

(a) Start position

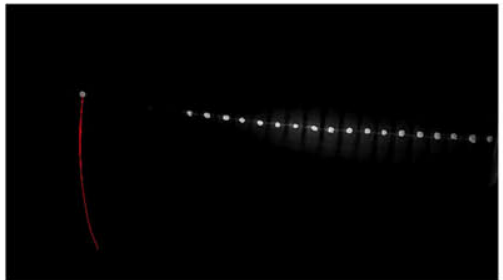

(c) Maximum Height

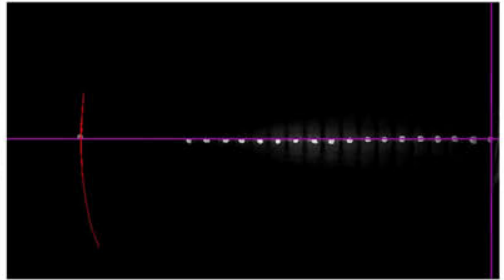

(b) Mid Point

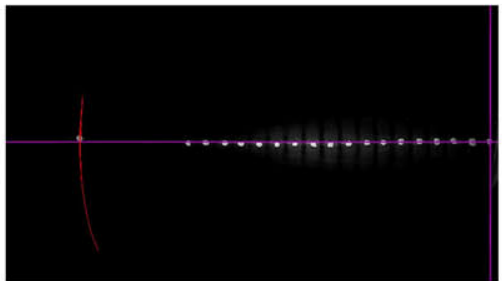

(d) End Position

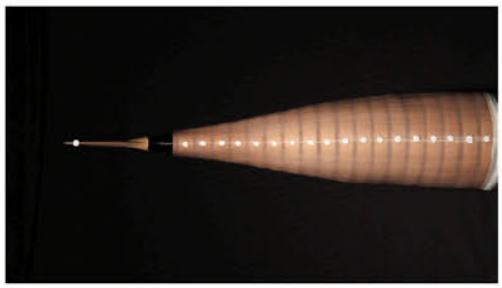

(e) Reference Fish view

Fig. 4. Free Vibration analysis of the backbone. The white spots are the marks for the tracking, the red line is the trajectory of the tail.

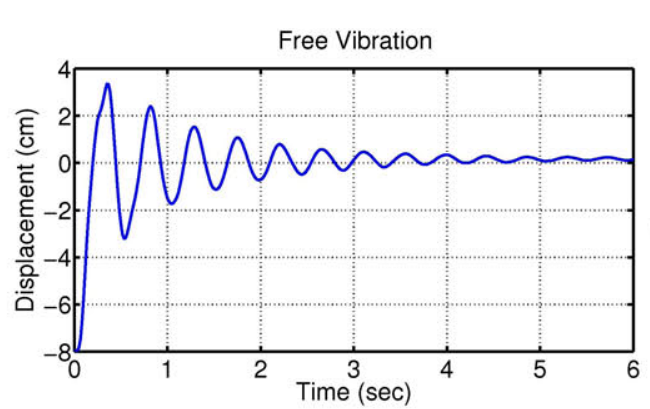

(a) Free Vibration (Displacement y axis)

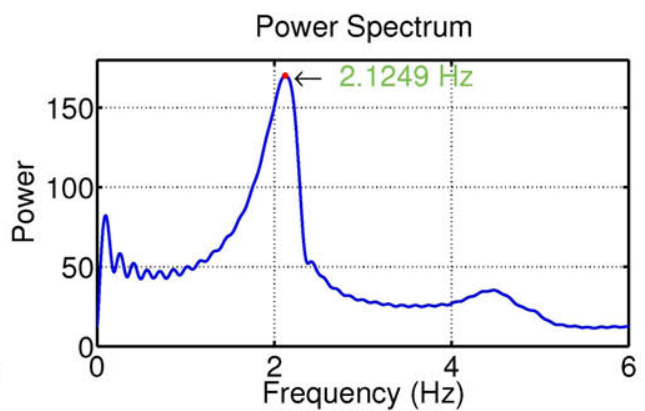

(b) Power Spectrum

Fig. 5. Experimental Results. The Natural frequency obtained was $2.1249 \mathrm{~Hz}$

\section{Conclusions}

In this work, we proposed the use of a theoretical model to find the Natural frequency of a Fish-like Robot with distributed masses along a flexible, continuous and non-uniform backbone. The Theoretical model proposed can be used for the beams with a large number of sections and capable of analyse the non-uniform beams with any variation in the cross 


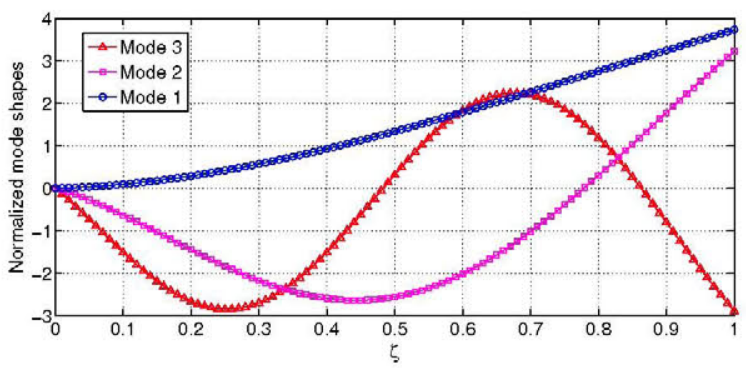

Fig. 6. First three modes of robot-fish with nineteen equally spaced similar concentrated masses.

section and moment of inertia. A comparison with an exact method for a set of cases where this could be applied assessed the goodness of the proposed method.

Comparing the data obtained experimentally for a physical prototype we could confirm that our method can effectively be used to analyse the free vibration in beams whose section is an arbitrary function, and with distributed masses.

Note the small variation of 0.2519 between the theoretical value (1.873) an the one obtained experimentally (2.1249). This is due to the flexible skin, whose effect was not considered. Adding the effects of the skin in the theoretical model will be object of future work.

\section{References}

1. Bert, C.W., Malik, M. (1996). Differential quadrature method in computational mechanics: A review. Applied Mechanics Reviews, 49, 1-28.

2. Youngsu Cha, Matteo Verotti, Horace Walcott, Sean D Peterson and Maurizio Porfiri, Energy harvesting from the tail beating of a carangiform swimmer using ionic polymermetal composites, Bioinspiration \& Biomimetics Volume 8 Number 3, 2013.

3. Chen, Y. (1963). On the vibration of beams or rods carrying a concentrated mass. Journal of Applied Mechanics, 30, 310-311.

4. W. Coral et al. Smart Actuation and Sensing Systems - Recent Advances and Future Challenges Chapter 3, SMA-Based Muscle-Like Actuation in Biologically Inspired Robots: A State of the Art Review (INTECH, 2012) 53-82

5. De Rosa, M.A., Franciosi, C., \& Maurizi, M.J. (1955). On the dynamics behavior of slender beams with elastic ends carrying a concentrated mass, Computers and Structures, 58, 11451159 .

6. Du, H., Lim, M.K., Lin, \& N.R. (1994). Application of generalized differential quadrature method to structural problems. International Journal for Numerical Methods in Engineering, 37, 1881-1896.

7. Du, H., Lim, M.K., \& Lin, N.R. (1995). Application of generalized differential quadrature to vibration analysis. Journal of Sound and Vibration, 181, 279-293.

8. Gurgoze, M. (1984). A note on the vibrations of restrained beams and rods with point masses. Journal of Sound and Vibration, 96, 461-468.

9. Gurgoze, M. (1985). On the vibration of restrained beams and rods with heavy masses. Journal of Sound and Vibration, 100, 588-589.

10. Kaneko, T. (1975). On Timoshenkos correction for shear in vibrating beams. Journal of Physics D: Applied Physics, 8, 1928-1937.

11. Karami, G., \& Malekzadeh, P. (2002). A new differential quadrature methodology for beam analysis and the associated differential quadrature element method. Computer Methods in Applied Mechanics and Engineerig,191, 3509-3526. 
12. Laura, P., Maurizi, M.J., \& Pombo, J.L. (1975). A note on the dynamics analysis of an elastically restrained-free beam with a mass at the free end. Journal of Sound and Vibration, 41, 397-405.

13. Laura, P., Verniere de Irassar, P.L., \& Ficcadenti, G.M. (1983). A note on transverse vibration of continuous beams subjected to an axial force and carrying concentrated masses, Journal of Sound and Vibration, 86, 279-284.

14. Lee, S.Y., \& Lin, S.M. (1995). Vibration of elastically restrained non-uniform Timoshenko beams. Journal of Sound and Vibration, 183, 403-415.

15. Lin, R.M., Lim, M.K., \& Du, H. (1994). Deflection of plates with nonlinear boundary supports using generalized differential quadrature. Computer and Structures, 53, 993-999.

16. Liu, W.H., Wu, J.R., \& Huang, C.C. (1988). Free vibrations of beams with elastically restrained edges and intermediate concentrated masses. Journal of Sound and Vibration, $122,193-207$.

17. Rao, G.V., Saheb, K.M., \& Janardhan, G.R. (2006), Fundamental frequency for large amplitude vibrations of uniform Timoshenko beams with central point concentrated mass using coupled displacement field method. Journal of Sound and Vibration, 298, 221-232.

18. C. Rossi, W. Coral et al., Bending continuous structures with SMAs: a novel robotic fish design, Bioinspiration \& Biomimetics 6, (2011) 15

19. C. Rossi, W. Coral et al., A Motor-less and Gear-less Bio-mimetic Robotic Fish Design, 2011 IEEE International Conference on Robotics and Automation (2011)

20. Timoshenko, S., Young, D.H., \& Weaver, W. (1974). Vibration problems in engineering. Wiley, New York. 